\title{
Volume Charge Density in Most General Lorentz Transformation
}

\author{
S. A. Bhuiyan*, A. R. Baizid \\ ${ }^{1}$ Department of Business Administration, Leading University, Sylhet-3100 \\ Received 28 March 2016, accepted in final revised form 13 June 2016
}

\begin{abstract}
Lorentz Transformations generally describe the transformations for observations between mechanical phenomenon systems in relative motion. We all know that the electrical charge of associate isolated system is relativistically invariant. We have studied the volume charge density in Special and Most General Lorentz Transformations. If one frame moves on $\mathrm{x}$-axis then we will notice this in Special Lorentz Transformation. On the other hand if the motion of the moving frame is not on the $\mathrm{x}$-axis relative to the rest frame however the motion is on any arbitrary direction then we will notice this formula for the Most General Lorentz Transformation. We also investigated the changes of the volume charge density of moving system in terms of that of rest system in Most General Lorentz Transformations at different angles and velocities.
\end{abstract}

Keywords: Volume Charge Density (VCD); Special Lorentz Transformation (SLT); Most General Lorentz Transformation (MGLT).

(c) 2016 JSR Publications. ISSN: 2070-0237 (Print); 2070-0245 (Online). All rights reserved. doi: http://dx.doi.org/10.3329/jsr.v8i3.27133

J. Sci. Res. 8(3), 259-265 (2016)

\section{Introduction}

The charge density is mainly a part of the concept of electromagnetism and it tells how much charge is present in a given length, area or volume. Therefore we can say, volume charge density tells specifically about how much electric charge is present in a given volume. It has been well established experimentally that the Lorentz Transformations provide an accurate translation of space and time measurements from one inertial reference frame regard to another. Rafiq and Alam [1] have derived the transformation equation of surface charge density in mixed number Lorentz Transformation. Bhuiyan et al. [2] have derived the transformation equation of surface charge density in different types of Lorentz Transformations. We choose a cube to find out the formula of volume charge density in different types of Lorentz Transformatons. In Lorentz Tranformations length is contracted. So the volume charge density will be

* Corresponding author: aktersabia@yahoo.com 
different in different types of Lorentz Transformations. In this paper we have derived the transformation equations in Most General Lorentz Transformations when the line of motion is not aligned with any of the co-ordinate axis.

\subsection{Special Lorentz Transformation}

Let us consider two inertial frames of references $S$ and $S^{\prime}$, where the frame $S$ is at rest and the frame $S^{\prime}$ is moving along x-axis with the velocity $\vec{v}$ with respect to the $S$ frame. The space and time coordinate of $S$ and $S^{\prime}$ are $(x, y, z, t)$ and $\left(x^{\prime}, y^{\prime}, z^{\prime}, t\right)$ respectively. The relation between the coordinates of $S$ and $S^{\prime \prime}$ which is called the Special Lorentz Transformations [1-7], can be written as,

$$
\begin{aligned}
& x^{\prime}=\gamma(x-\vec{v} t) \\
& y^{\prime}=y \\
& z^{\prime}=z \\
& t^{\prime}=\gamma(t-\vec{v} x)
\end{aligned}
$$

Where $\gamma=\frac{1}{\sqrt{1-\frac{v^{2}}{c^{2}}}} \quad$ and $c=1$

The inverse Lorentz Transformations [1-7] can be written as

$$
\begin{aligned}
& x=\gamma\left(x^{\prime}+\vec{v} t^{\prime}\right) \\
& y=y^{\prime} \\
& z=z^{\prime} \\
& t=\gamma\left(t^{\prime}+\vec{v} x^{\prime}\right)
\end{aligned}
$$

Where $\gamma=\frac{1}{\sqrt{1-\frac{v^{2}}{c^{2}}}}$ and $c=1$

\subsection{Most General Lorentz Transformation}

When the velocity $\vec{v}$ of $S^{\prime}$ with respect to $S$ is not along the x-axis, i.e. the velocity $\vec{v}$ has three components $v_{x}, v_{y}, v_{z}$ then the relation between the coordinates of $S$ and $S^{\prime}$, which is called the Most General Lorentz Transformations [1-3] can be written as.

$$
\vec{x}^{\prime}=\vec{x}+\vec{v}\left[\left\{\frac{\vec{x} \cdot \vec{v}}{v^{2}}(\gamma-1)\right\}-t \gamma\right]
$$


$t^{\prime}=\gamma(t-\vec{v} \vec{x})$

Where $\gamma=\frac{1}{\sqrt{1-\frac{v^{2}}{c^{2}}}}$ and $c=1$

The inverse Most General Lorentz Transformations [1-3] can be written as

$$
\begin{aligned}
& \vec{x}=\vec{x}^{\prime}+\vec{v}\left[\left\{\frac{\vec{x} \cdot \vec{v}}{v^{2}}(\gamma-1)\right\}+t^{\prime} \gamma\right] \\
& t=\gamma\left(t^{\prime}+\vec{v} \cdot \vec{x}^{\prime}\right)
\end{aligned}
$$

\section{Volume Charge Density}

Volume charge density means the amount of electric charge per unit volume. It is denoted by $\rho$.

$$
\therefore \rho=\frac{q}{V}
$$

It is measured by coulombs per cubic meter $\left(\mathrm{c} / \mathrm{m}^{3}\right)$. There are positive as well as negative charges, so the charge density can take on negative values. It depends on position. We know that the charge on the electron or proton is the minimum, called the elementary charge $e\left(1.6 \times 10^{-19}\right.$ coul $)$. The electric charge is discrete which may be determined by counting the number of elementary charged particles. As the total number of elementary charges do not depend on the state of the motion of the observer, we may conclude that the electric charge is relativistically invariant. Based on the conclusion we want to calculate the transformation equation for the volume charge density $\rho[1,2]$.

\section{Transformation of Volume Charge Density}

\subsection{Transformation of volume charge density in Special Lorentz Transformation}

Let us consider two systems $S$ and $S^{\prime}, S^{\prime}$ is moving with uniform velocity $\vec{v}$ relative to $S$ along negative direction of $\mathrm{x}$-axis as shown in Fig 1 . Let there be a stationary sheet of uniform charge density $+\rho\left(\mathrm{c} / \mathrm{m}^{3}\right)$ at rest in system $S$ having one edge parallel to $\mathrm{X}$-axis. Let there be a cube of side $L_{0}$ and placed parallel to $x-y$ plane. The observer in system $S^{\prime}$ will observe that the cube is moving with the velocity $\vec{v}$ along (+ve) x-axis.

We have the transformation relation of volume charge density from the principle of invariance of charge which states, 'the total electric charge in an isolated system is relativistically invariant'. The total charge as observed in the system $S$ is

$$
Q_{o}=L^{3} \rho
$$




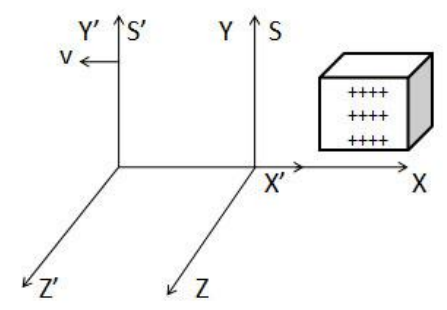

Fig. 1 . The system $S^{\prime}$ is moving along $x$-axis with velocity $\mathbf{v}$ relative to the system $S$.

The observer in system $S^{\prime}$ will notice that the side of the cube along x-axis has been contracted from $L_{0}$ to $L$, where

$$
L=\frac{L_{0}}{\gamma}=\left(\sqrt{1-\frac{v^{2}}{c^{2}}}\right) L_{0}
$$

As the length is contracted only along $\mathrm{x}$-axis, the charge as observed by the observer in the system $S^{\prime}$ is

$$
Q^{\prime}=\left(\sqrt{1-\frac{v^{2}}{c^{2}}}\right) \cdot L_{0}^{3} \rho^{\prime}
$$

Where $\rho^{\prime}$ is the volume charge density in system $S^{\prime}$. According to the principle of conservation of charge

$$
\begin{array}{r}
Q^{\prime}=Q_{0} \\
\text { or, }\left(\sqrt{1-\frac{v^{2}}{c^{2}}}\right) \cdot L_{0}^{3} \rho^{\prime}=L_{0}^{3} \rho \\
\therefore \rho^{\prime}=\frac{\rho}{\sqrt{1-\frac{v^{2}}{c^{2}}}}=\gamma \rho
\end{array}
$$

This equation represent the transformation equation for the volume charge density in Special Lorentz transformation.

\subsection{Transformation of volume charge density in Most General Lorentz Transformation}

Let us consider two systems $S$ and $S^{\prime}, S^{\prime}$ are moving with uniform velocity $\vec{v}$ relative to $S$ along any arbitrary direction as shown in Fig 2 . Thus the velocity $\vec{v}$ has three 
components $v_{x}, v_{y}$ and $v_{z}$. Let there be a stationary sheet of uniform charge density $+\rho\left(\mathrm{c} / \mathrm{m}^{3}\right)$ at rest in system $S$ having one edge parallel to x-axis. Let the sheet be a cube of side $\mathrm{L}_{0}$ and placed parallel to x-y plane. The observer in system $S^{\prime}$ will observe that the cube is moving with the velocity $\vec{v}$ in opposite direction of the system $S$.

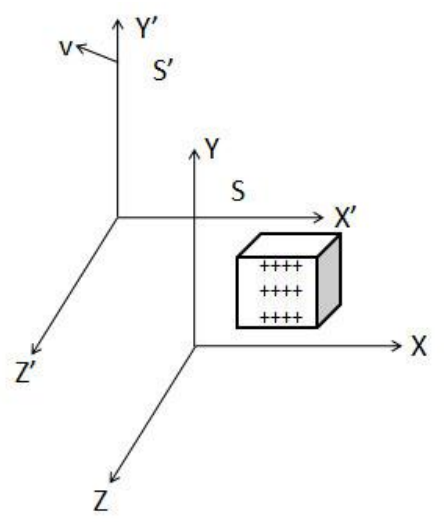

Fig. 2. The system $S^{\prime}$ is moving in $x-y$ plane with velocity $\mathbf{v}$ relative to the system $S$.

If $L_{0}$ is the length of the cubed charge sheet in $S$ then the length contraction in the moving frame for the most general Lorentz transformation [8] can be written as

$$
\begin{aligned}
& \vec{L}_{o}=\vec{L}+\frac{\vec{v}}{v^{2}}\left[x_{2}^{\prime} v \cos \theta-x_{1}^{\prime} v \cos \theta\right](\gamma-1) \\
& \text { or } \vec{L}_{o}=\vec{L}+\frac{\bar{v}}{v} L \cos \theta(\gamma-1) \\
& \text { or } \quad L_{o}^{2}=\left[\vec{L}+\frac{\vec{v}}{v} L \cos \theta(\gamma-1)\right] \cdot\left[\vec{L}+\frac{\vec{v}}{v} L \cos \theta(\gamma-1)\right] \\
& \quad=L^{2}\left[1+2 \cos ^{2} \theta(\gamma-1)+\cos ^{2} \theta(\gamma-1)^{2}\right] \\
& L_{x}^{2}=\frac{L_{o}^{2}}{\left[1+2 \cos ^{2} \theta(\gamma-1)+\cos ^{2} \theta(\gamma-1)^{2}\right]} \\
& L_{x}^{2}=\frac{L_{o x}^{2}+L_{o y}^{2}+L_{o z}^{2}}{\left[1+2 \cos ^{2} \theta(\gamma-1)+\cos ^{2} \theta(\gamma-1)^{2}\right]} \\
& L_{y}^{2}=\frac{L_{o x}^{2}}{\left[1+2 \cos ^{2} \theta(\gamma-1)+\cos ^{2} \theta(\gamma-1)^{2}\right]}
\end{aligned}
$$




$$
L_{z}^{2}=\frac{L_{o z}^{2}}{\left[1+2 \cos ^{2} \theta(\gamma-1)+\cos ^{2} \theta(\gamma-1)^{2}\right]}
$$

The total charge observed by an observer in system $S^{\prime}$ is

$$
\begin{aligned}
& Q^{\prime}=L_{x} L_{y} L_{z} \rho \\
& =\sqrt{\frac{L_{o x}^{2}}{\left[1+2 \cos ^{2} \theta(\gamma-1)+\cos ^{2} \theta(\gamma-1)^{2}\right]}} \times \sqrt{\frac{L_{o z}^{2}}{1+2 \cos ^{2} \theta(\gamma-1)+\cos ^{2} \theta(\gamma-1)^{2}}} \\
& \quad \times \sqrt{\frac{L_{o z}^{2}}{\left[1+2 \cos ^{2} \theta(\gamma-1)+\cos ^{2} \theta(\gamma-1)^{2}\right]}} \rho \\
& \therefore Q^{\prime}=\frac{L_{o}^{3}}{\left[1+2 \cos ^{2} \theta(\gamma-1)+\cos ^{2} \theta(\gamma-1)^{2}\right]^{\frac{3}{2}}}
\end{aligned}
$$

According to the principle of conservation of charge

$Q^{\prime}=Q_{o}$

Using equation (19) and (13) we can write

$$
\begin{aligned}
& \frac{L_{o}^{3}}{\left[1+2 \cos ^{2} \theta(\gamma-1)+\cos ^{2} \theta(\gamma-1)^{2}\right]^{\frac{3}{2}}} \rho^{\prime}=L_{o}^{3} \rho \\
& \therefore \rho^{\prime}=\left[1+2 \cos ^{2} \theta(\gamma-1)+\cos ^{2} \theta(\gamma-1)^{2}\right]^{\frac{3}{2}} \times \rho
\end{aligned}
$$

This equation represents the transformation equation for the volume charge density when the system $S^{\prime}$ is moving along any arbitrary direction.

Table1. Transformation equation for VCD in SLT and MGLT.

\begin{tabular}{lll}
\hline & SLT & MGLT \\
\hline $\begin{array}{l}\text { Length } \\
\text { contraction }\end{array}$ & $L_{0}=\gamma L$ & $\vec{L}_{o}=\vec{L}+\frac{\bar{v}}{v} L \cos \theta(\gamma-1)$ \\
$\begin{array}{l}\text { Volume } \\
\text { charge density }\end{array}$ & $\rho^{\prime}=\gamma \rho$ & $\therefore \rho^{\prime}=\left[1+2 \cos ^{2} \theta(\gamma-1)+\cos ^{2} \theta(\gamma-1)^{2}\right]^{\frac{3}{2}} \times \rho$ \\
\hline
\end{tabular}

Table 2. Numerical values of VCD of moving system in terms of that of rest system in SLT and MGLT at different angles and velocities.

\begin{tabular}{llll}
\hline Parameter & & SLT & MGLT \\
\hline$\theta=30^{\circ}$ & $\mathrm{v}=.3 \mathrm{c}$ & --- & $0.4045 \rho$ \\
& $\mathrm{v}=.5 \mathrm{c}$ & --- & $0.2682 \rho$
\end{tabular}




\begin{tabular}{llll}
\hline Parameter & SLT & MGLT & Parameter \\
\hline$\theta=45^{\circ}$ & $\mathrm{v}=.3 \mathrm{c}$ & --- & $0.4355 \rho$ \\
& $\mathrm{v}=.5 \mathrm{c}$ & --- & $0.3350 \rho$ \\
$\theta=60^{\circ}$ & $\mathrm{v}=.3 \mathrm{c}$ & --- & $0.4670 \rho$ \\
& $\mathrm{v}=.5 \mathrm{c}$ & --- & $0.4120 \rho$ \\
\hline
\end{tabular}

* Calculations were carried out taking c as unity

\section{Conclusion}

The transformation formula for volume charge density in Special and Most general Lorentz transformation are illustrated in Table 1. The numerical values of the volume charge densities of the moving system in terms of a system at rest for most general Lorentz transformation were calculated as shown in Table 2. From Table 2 it is observed that for the same angle $(\theta)$ between the system $S$ and $S^{\prime}$, the value of the volume charge density of the moving system $\left(\rho^{\prime}\right)$, decreases with increasing velocity $\vec{v}$ of the moving system and for the same velocity of the moving system $\rho^{\prime}$ increases with increasing the angle $\theta$.

\section{References}

1. S. B. Rafiq and M. S. Alam, Sri Lankan J. Phys. 13(1), 17 (2012).

2. S. A. Bhuiyan and A. R. Baizid, Appl. Math. 5(3), 57 (2015).

3. A. R. Baizid and M. S. Alam, Am. J. Math. Stat. 2(5), 153 (2012). http://dx.doi.org/10.5923/j.ajms.20120205.08

4. R. Resnick, Introduction to Special Relativity (Wiley astern Limited, 1994).

5. C. Moller, The Theory of Relativity (Oxford Univ. Press, London, 1972).

6. S. Prakash, Relativistic Mechanics (Progati Prakashani, New Delhi, 1993-94)

7. M. R. Spiegel, Theory and Problems of Vector Analysis and An Introduction to Tensor Analysis, Schaum Outline Series, $2^{\text {nd }}$ edition (Mc Graw-Hill Book Company, 2009).

8. M. S. Alam and K. Begum, Jahangirnagar Phys. Stud. 15, 89 (2009). 